AIAA 2001-1138

A Simplified Model of ARIS for Optimal Controller Design

G. Beech

NASA Marshall Space Flight Center

Huntsville, AL

R. David Hampton

University of Alabama in Huntsville

Huntsville, AL 


\title{
A SIMPLIFIED MODEL OF ARIS FOR OPTIMAL CONTROLLER DESIGN
}

\author{
Geoffrey S. Beech* \\ NASA, Marshall Space Flight Center, Huntsville Alabama \\ R. David Hampton $\mathbf{t}$ \\ University of Alabama in Huntsville, Huntsville, Alabama
}

\begin{abstract}
Many space-science experiments require active vibration isolation. Boeing's Active Rack Isolation System (ARIS) isolates experiments at the rack (vs. experiment or sub-experiment) level, with multiple experiments per rack. An ARIS-isolated rack typically employs eight actuators and thirteen umbilicals; the umbilicals provide services such as power, data transmission, and cooling.

Hampton, et al., used "Kane's method" to develop an analytical, nonlinear, rigid-body model of ARIS that includes full actuator dynamics (inertias). This model, less the umbilicals, was first implemented for simulation by Beech and Hampton; they developed and tested their model using two commercial-off-the-shelf (COTS) software packages. Rupert, et al., added umbilicaltransmitted disturbances to this nonlinear model. Because the nonlinear model, even for the untethered system, is both exceedingly complex and "encapsulated" inside these COTS tools, it is largely inaccessible to ARIS controller designers.

This paper shows that ISPR rattle-space constraints and small ARIS actuator masses permit considerable model simplification, without significant loss of fidelity. First, for various loading conditions, comparisons are made between the dynamic responses of the nonlinear model (untethered) and a truth model. Then comparisons are made among nonlinear, linearized, and linearized reduced-mass models. It is concluded that these three models all capture the significant system rigid-body dynamics, with the third being preferred due to its relative simplicity.
\end{abstract}

\section{INTRODUCTION}

Acceleration levels currently achievable on the International Space Station (ISS) exceed those required by many space-science experiments. (See, for example, Delombard, et al., 1997'). Various active isolation systems have been built to address the need for microgravity isolation. The first was called STABLE

\footnotetext{
* Member AIAA

$\dagger$ Associate Professor of Mechanical Engineering, Member AIAA
}

("Suppression of Transient Accelerations By Levitation"), which uses six independently controlled Lorentz actuators to levitate and isolate at the experiment level (Edberg, et al., 1996 ${ }^{2}$ ). STABLE was successfully flight-tested on STS-73 (USML-02) in October 1995. NASA's Marshall Space Flight Center is currently developing a second-generation experiment-level isolation system (g-LIMIT: "Glovebox Integrated Microgravity Isolation Technology"), building on the technology developed for STABLE (Whorton, 1998 ${ }^{3}$ ). This compact system will isolate microgravity payloads in the Microgravity Science Glovebox (MSG).

For the ISS, the Boeing Corporation has developed the Active Rack Isolation System (ARIS), which, in contrast to these above two experiments, isolates at the International Standard Payload Rack (ISPR) level. Instead of using magnetic levitation, ARIS employs a set of eight voice-coil (Lorentz) actuators strategically mounted between the exterior of each ARIS-outfitted ISPR and the ISS, as shown in Figure 1.

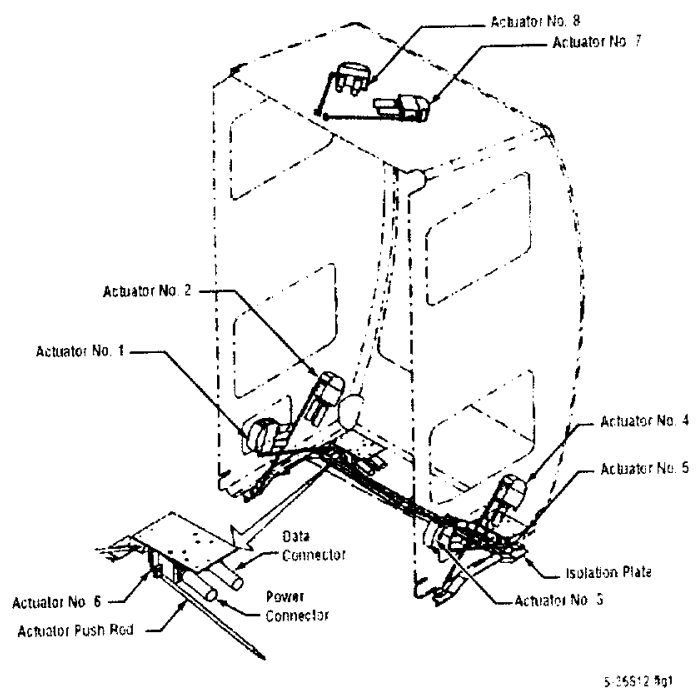

Figure 1. ARIS-Outfitted ISPR

The separation of the actuators around the ISPR increases the torque authority, and therefore reduces the weight and power requirements, of the system. One disadvantage of ARIS, compared to a magnetically 
levitated system, is the additional complexity in the mathematical description necessary for controller design.

In order to provide for model-based controller design, one needs, first, an appropriate physical isolation device; and second, a mathematical model for the system dynamics of that isolator. In the present case, ARIS is the physical device; this paper adresses the development of an appropriate mathematical model of ARIS, to facilitate the development of a suitable controller. The linearized equations of motion for a controlled dynamical system can be written in the following descriptor form:

$$
[M]\{\underline{\dot{x}}\}=[A]\{\underline{x}\}+[B]\{\underline{i}\}+[E]\{\underline{d}\},
$$

where system matrices $[M]$ and $[A]$ represent the physics of the system, and control input matrix $[B]$ describes the manner in which the control input is applied. The control vector $\underline{i}$ is the vector of control currents, applied to the Lorentz coils. The disturbance vector $\underline{d}$ accounts for indirect disturbances (i.e., transmitted indirectly from the ISS via the actuators or umbilicals) and direct disturbances (i.e., directly applied to the ISPR). $[E]$ is the system disturbance input matrix. (For anticipated disturbance levels, see NASA Specification Number SSP41000, Rev. D., 1995 ${ }^{4}$ ).

\section{DESCRIPTION OF ARIS}

The total dynamical system $\tilde{S}$ consists of the stator $S$ (ISS and the integral frame, from the motion of which ARIS isolates the ISPR), the flotor $F$ (the ISPR), the eight electromechanical actuator assemblies, and the umbilicals. The ISPR is connected to the ISS by eight

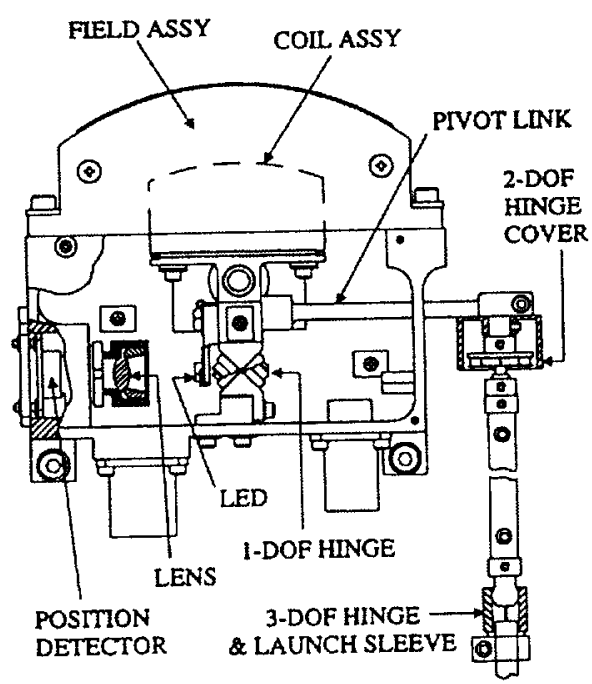

Figure 2. Actuator Assembly Diagram actuator assemblies, and by a variable number (typically thirteen) of umbilicals. The actuator assemblies are used to apply forces for attenuation of indirect and direct disturbances, using sensed payload inertial accelerations and relative positions.

Each actuator assembly consists of a rotary-type Lorentz (voice-coil) actuator with a position sensor, an arm, and a push-rod, as shown in Figure 2. The ARIS actuators have been designed for negligible static and kinetic friction. Each actuator arm is connected to the ISPR through an associated cross-flexure, a joint with a single rotational degree of freedom. The other end of each arm is connected to its respective push-rod through an upper stinger, a joint with two rotational degrees of freedom (in bending). The opposite end of each pushrod is connected to the ISS through a lower stinger, to allow three rotational degrees of freedom (two in bending, one in torsion) with respect to the ISS. Each actuator joint is modeled as a spring with no mass.

For modeling purposes, the ISS, the ISPR, the actuator arms and the push-rods are considered to be rigid bodies, with mass centers at points $S^{*}, F^{*}, A_{i}^{*}$, and $P_{i}^{*}$, respectively. The superscript * indicates the mass center of the indicated rigid body; the subscript $i$ corresponds to the $i^{\text {th }}$ actuator $(i=1, \ldots, 8)$. All springs (cross-flexures and stingers) are assumed to be relaxed when the ISPR is centered in its rattlespace (the "home position"). Umbilical stiffnesses and dampings are not included in this paper; they are treated separately by Rupert and Hampton.

\section{METHODOLOGY}

G. Beech and D. Hampton ${ }^{5}$ previously reported using the method of Thomas Kane ${ }^{6}$ to develop a linearized, analytical, rigid-body model of ARIS. Model development was initially accomplished directly by hand (rather than by the more difficult task of linearizing a full nonlinear model), and checked subsequently by symbolic-manipulation computer software. The linearized model includes the following inputs: (1) direct actuator control forces (i.e., applied directly to the ISPR via the actuator), (2) direct disturbance forces (i.e., acting directly on the ISPR), (3) indirect, actuator-transmitted disturbances (i.e., transmitted from the ISS along the eight actuators to the ISPR), and (4) umbilical-transmitted disturbances, under the assumption of diagonal umbilical-stiffness and -damping matrices. The ARIS actuator angles, six per actuator, were chosen as coordinates.

Beech and Hampton subsequently completed, calibrated, and tested [using commercial-off-the-shelf (COTS) software] a corresponding nonlinear, analytical, rigid-body model for the untethered system. This nonlinear "Kane's model" was desired for the following 
reasons: (1) to simulate the full, nonlinear, rigid-body system; (2) to determine the range, and degree, of fidelity of the linearized model; and (3) to seek the simplest obtainable high-fidelity, dynamical model which would be suitable for controller design. A nonlinear model was first developed for the simpler, untethered system. (The tethers were included later, as a separate task. ${ }^{7}$ ) Even without tethers the ensuant nonlinear model is exceedingly complex. Further (in consequence), it is, to a great extent, "encapsulated" inside the COTS tools in a way that made its details largely inaccessible to controller designers. In addition, the matrices $[M]$ and $[A]$ described above are large (54 $x$ 54), nonlinear, and generally not well-suited (due to their great complexity) for entry into MATLAB, or other standard controller-design software tools. Fortunately, ARIS' inertia characteristics and limited range of motion permit model-simplification, so that a more manageable representation of the system dynamics is possible.

It would have been desirable to validate the ARIS model (in the form of Eq. 1) by first testing the actual ARIS hardware in a near-microgravity (milli-g) environment and then using those results as a basis for model evaluation. However, because such testing of ARIS can only be accomplished on-orbit, computersimulation models were chosen to achieve a high degree of confidence in the nonlinear analytical model, in anticipation of on-orbit tests.

Two complementary kinds of high-fidelity computer-simulation models were developed: kinematical and dynamical. The kinematical model of ARIS is a nonlinear, 18-rigid-body model (ISS, plus ISPR, plus eight actuator arms, plus eight actuator pushrods) based on high-fidelity CAD models; it computes and displays (pictorially and numerically) the configuration of the eight actuators for arbitrary positions of the ISPR. Measurements from the supporting CAD models provide numerical values (e.g., geometric lengths, inertias, position coordinates, and actuator angles) for use in the dynamical models. The dynamical models are 18-rigid-body analytical models that, when properly initialized and calibrated, can compute ARIS actuator-angles and -angular rates for various force and moment test inputs. The actuatorangle outputs from these models can be compared to data from the kinematical model at any stage (snapshot) in a dynamical simulation, to ensure proper incorporation of the configuration constraints. The basic dynamical model is a nonlinear model with full masses and inertias. Various simplifications to this model produced other dynamical models of ARIS.

A two-body baseline or truth model of the ARIS dynamics was also developed, to provide a benchmark for comparison against the full 18-rigid-body models. The truth model represents an ISPR in zero gravity, without any actuators or umbilicals, having the same mass properties as in the basic nonlinear dynamical model. The six degrees of freedom used to specify the position and orientation of the ISPR are translation along, and rotation about, the three ISS coordinate axes.

The system dynamics of the basic dynamical (18body, nonlinear, full-inertia) model are compared to the system dynamics of the truth (two-body) model. This comparison is accomplished by reducing the actuator spring constants (internal actuator forces) of the nonlinear model to negligible levels, and applying a common set of test inputs directly to the ISPR of both models. This gives a good comparison between the two models for motion of the ISPR within its rattlespace.

Beginning with the nonlinear model two assumptions can be made to simplify it, for easier use with traditional controller-design tools (such as MATLAB), without sacrificing model fidelity. First, because the motion of an ARIS-equipped ISPR is limited, the actuator angles can be assumed to remain small. This permits linearization using small angle approximations. Second, because the masses of the arms and push-rods of the ARIS actuators represent only a small fraction of the overall ISPR mass, their inertial forces can be neglected. It is important to note that with this latter simplification only the inertial forces of the actuator arms and push-rods are neglected-all of the actuators' applied and internal forces are retained. These simplifications are treated further in the following sections.

\section{SOFTWARE TOOLS}

The development and validation of the basic dynamical model were achieved using two CommercialOff-The-Shelf (COTS) software tools: Delmia's (formerly Deneb Robotics) Envision and OnLine Dynamics' AUTOLEV.

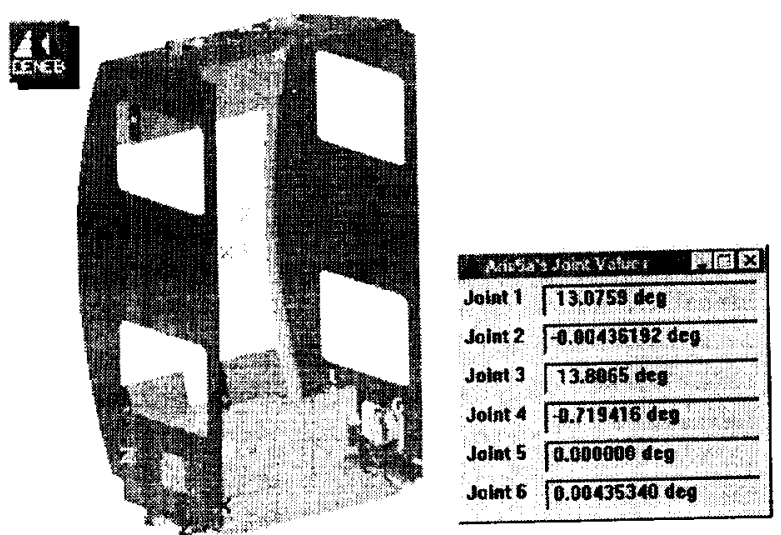

Figure 3. Envision Model of ARIS 
Envision is a robotics software package developed for the automotive industry that employs threedimensional CAD models to facilitate forward and inverse kinematics analyses. Once CAD models of each of the rigid bodies of ARIS are translated into Envision, coordinate systems representing the attachment locations are added. The ARIS actuators are then built up individually in a parent-child fashion from the CAD models, and an algorithm for an inverse kinematics solution is applied to each. Next all of the actuators, the ISS, and the ISPR are appropriately combined, and the system is activated in a common CAD environment. A user can then specify the configuration of a single input actuator, and the remaining seven actuators will be moved to their corresponding configurations, as determined by their respective inverse kinematics algorithms and the constraints of the system. Then the configuration information for each of the actuators can be compared to the configuration specified by the AUTOLEV simulations.

AUTOLEV is a DOS-based interpreter that is designed, in general, to solve vector-based mathematical problems and, in particular, to solve dynamics problems using "Kane's method"6,8. Because AUTOLEV is an interpreter, all of the commands entered for a particular session are stored in memory and can be written to a file. Alternatively, a user can develop a text file that contains the commands to be read by AUTOLEV, for a particular session, as if the commands had been entered manually. The output from AUTOLEV is a $C$ program. This program, when compiled, linked, and run, creates a DOS executable file which, in turn, creates a data file of the specified output for a given simulation run. There is a close correspondence between the linearized analytical ARIS model of Equation 1 and the nonlinear AUTOLEV model, because the underlying developments for both use Kane's method.

\section{NONLINEAR ANALYTIC MODEL}

Using Kane's method, Hampton and Beech developed an analytical model of the ARIS dynamics that incorporates the actuator dynamics and utilizes coordinate systems based on the ARIS actuators ${ }^{5}$. This 18-body model consists of fifty-four equations: fortytwo constraint equations, six kinematical equations, and six dynamical equations. If one considers an ARISoutfitted ISPR moving relative to the ISS, the ISPR motion can be fully described in terms of six degrees of freedom. (The ISS motion can be considered to be translational only, assuming very small ISS angular velocities and angular rates, and to provide disturbance inputs to a six-degree-of-freedom system.) These six degrees of freedom are commonly, although not necessarily, characterized by translations along, and rotations about, the (ISS) $x$-, $y$ - and $z$-axis directions.

With the ARIS system, however, there are eight six-degree-of-freedom actuators attached between the ISPR and the ISS. These actuators represent forty-eight degrees of freedom, many more than necessary for describing the configuration of an ARIS-outfitted ISPR. Indeed, the specification of the configuration of any single actuator completely describes the configuration of the ISPR relative to the ISS. Therefore, the fortyeight degrees of freedom can be divided into six independent, and forty-two dependent, degrees of freedom.

Although ARIS contains only six independent degrees of freedom, as noted above, it is necessary to know the values and time-rates-of-change of all fortyeight degrees of freedom in order to describe the system dynamics. Therefore, one can select the forty-eight coordinates and the forty-eight time-rates-of-change of these coordinates as the ninety-six states of the system. Knowledge of these ninety-six states, as functions of time, is necessary and sufficient for knowledge of the dynamics of the 18-rigid-body system.

It follows that ninety-six equations are needed to solve the ninety-six unknowns of ARIS. The equations can be classified generally as kinematical, dynamical, and constraint equations. The kinematical equations simply express the time-rates-of-change of the coordinates as generalized speeds. Thus, the forty-eight kinematical equations represent this simple relationship:

$$
\dot{q}_{i}=u_{i}(\text { for } i=1, \ldots, 48)
$$

where $q_{i}$ represents the $i^{t h}$ coordinate; and $u_{i}$, the $i^{t^{t h}}$ generalized speed.

Forty-two of the ninety-six equations are holonomic constraint equations, which can be expressed most simply in non-holonomic form. Twenty-one of the equations represent the fact that each component of the angular velocity of the ISPR, regardless of the actuator through which it is specified, is a single quantity. Another twenty-one equations represent the fact that each component of the velocity of the center-of-mass of the ISPR (again, regardless of the actuator through which it is specified), is a single quantity. The fortytwo constraint equations can be expressed as follows:

$$
u_{r}=\sum_{s=1}^{6} A_{r s} u_{s}(\text { for } r=7, \ldots, 48),
$$

where the $u_{s}$ 's are the independent generalized speeds, the $u_{r}$ 's are the dependent generalized speeds, and the $A_{r s}$ 's are scalar coefficients derived from the constraint equations (Kane, T. and Levinson, D., 1995 ${ }^{6}$ ). 
The remaining six equations are the dynamical equations for ARIS. It is these six dynamical equations which are particularly complex for the ARIS system. Some of the factors contributing to their complexity are the number of rigid bodies in the system, the connecting springs, and the over-constrained nature of ARIS. Using the superscripts $I$ and $D$ to mean "independent" and "dependent," respectively, define the following:

$$
\begin{gathered}
\underline{q}^{\prime}=\left[\begin{array}{cccccc}
q_{1}^{1} & q_{2}^{1} & q_{3}^{1} & q_{4}^{1} & q_{5}^{1} & q_{6}^{1}
\end{array}\right]^{T}, \\
\underline{u}^{l}=\dot{q}^{\prime}, \\
\underline{u}^{D}=\dot{q}^{D},
\end{gathered}
$$

and

where the numerical superscripts and subscripts indicate the actuators and actuator angles (in some convenient order), respectively. The equations of motion for ARIS can now be expressed in the following descriptor form:

$$
\begin{gathered}
{\left[\begin{array}{lll}
I & O & O \\
O & I & O \\
O & O & M
\end{array}\right]\left\{\begin{array}{l}
\dot{q}^{I} \\
\dot{q}^{D} \\
\dot{u}^{I}
\end{array}\right\}=\left[\begin{array}{ccc}
O & O & I \\
O & O & N \\
K_{i} & K_{d} & C
\end{array}\right]\left\{\begin{array}{l}
\underline{q}^{I} \\
\underline{q}^{D} \\
\underline{u}^{l}
\end{array}\right\}} \\
+\left[\begin{array}{l}
O \\
O \\
B
\end{array}\right]\{\underline{i}\}+\left[\begin{array}{l}
O \\
O \\
E
\end{array}\right]\{\underline{d}\}
\end{gathered}
$$

The state vector consists of the 48 coordinates $\left\{\begin{array}{ll}q^{I} & q^{D}\end{array}\right\}^{T}$ and the six independent generalized speeds $\underline{u}^{l}$, where

$$
\underline{u}^{\prime}=\left[\begin{array}{llllll}
u_{1}^{1} & u_{2}^{1} & u_{3}^{1} & u_{4}^{1} & u_{5}^{1} & u_{6}^{1}
\end{array}\right]^{r} .
$$

The constant submatrices $[M],\left[K_{i} K_{d}\right]$ and $[C]$ are system mass, stiffness, and damping matrices, respectively; the $42 \times 6$ constant matrix $N$ comes from the constraint equations, where the $i j$ element $N_{i j}$ is $A_{r s}$, for $r=i+6$ and $s=j$. The symbols $I$ and $O$ represent, respectively, an identity matrix and a zero matrix of appropriate dimensions; the vector $\underline{i}$ contains the eight control currents to the Lorentz coils; and the vector $\underline{d}$ is the disturbance vector. The elements of input matrices $B$ and $E$ are time-varying, nonlinear functions of the coordinates.

As indicated previously, the nonlinear model above is prohibitively complex for symbolic entry into MATLAB or other traditional controller design software, and is presented here only in representative vector form. A computer-based, nonlinear, encapsulated, rigid-body, analytical model of ARIS (untethered) was successfully developed in AUTOLEV, however, and calibrated numerically using the Envision model.

\section{THE FREE-FLOA TING ISPR (TRUTH) MODEL}

A second dynamical model was developed in AUTOLEV to represent the system dynamics of an ISPR in zero gravity with characteristic mass properties, but without any actuators. This benchmark, or truth, model calculates the response in translation and rotation of the ISPR center of mass in response to test forces applied at various points on the ISPR. The coordinates used in this model are translation along, and rotation about, the ISS-fixed $x-, y$ - and $z$-axes. This model provides a basis for comparison, by simulation, of other more complex dynamical models, primarily because hardware validation would require resources beyond those currently available.

An advantage of this model is its simplicity; it is limited, however, in that it is useful for comparison purposes only when the dynamics of the actuators are negligible. This means, first, that the torsional springs at the lower and upper stingers and cross-flexures (for the full dynamical models against which it is being compared) must have negligible stiffnesses. Second, the actuators must not reach full-extension. The actuator spring constants are easily changed to negligible levels in the full dynamical models, by appropriately specifying their values in the AUTOLEV code. The full-extension constraints only come into play when the actuators would otherwise extend beyond their physical limitations. In the actual hardware, the ISPR displacements from the home position are limited to a half-inch rattle-space by a snubber system. In the dynamical simulation models there is no such system, however, and the motion must be limited by the disturbance input or the simulation duration.

\section{LINEARIZED ANALYTICAL MODEL}

With the nonlinear model of ARIS completed, linearization is relatively easy using AUTOLEV. The automatic linearization of the generalized active and generalized inertial forces is accomplished with a single AUTOLEV command. The arguments this command describe the order of the terms of a Taylor-series expansion, here the $0^{\text {th }}$ and $1^{\text {st }}$-order terms, and the arguments about which the Taylor-series expansion is to be performed. The result is a linearized form of the ARIS dynamics.

To realize fully the impact of linearization, one must appreciate the enormous complexity of the nonlinear dynamical equations. The six dynamical equations of ARIS are obtained using the following process. First, add the respective contributions of the 17 rigid bodies (the ISS is assumed for the present to be infinitcly massive or, equivalently, to have specified motion, and makes no contribution) to the set of 
holonomic generalized active and -generalized inertia forces, for each generalized speed (i.e., $r=1, \ldots, 48$ ) as defined using the two equations,

$$
\begin{array}{ll}
Q_{r}=\underline{v}_{r} \cdot \underline{R}+\underline{\omega}_{r} \cdot \underline{T}, \\
\text { and } Q_{r}^{*}=\underline{v}_{r} \cdot \underline{R}^{*}+\underline{\omega}_{r} \cdot \underline{T}^{*},
\end{array}
$$

respectively. The factors $\underline{v}_{r}$ and $\underline{\omega}_{r}$ are the partial derivatives of the velocity and angular velocity, repectively, of the affected point or body, with respect to each of the forty-eight generalized speeds. $\underline{R}^{*}$ and $T^{*}$ are the inertial force and inertial torque, respectively, produced by the motion and mass properties of the ARIS bodies. The holonomic generalized active force for the $r^{\text {th }}$ generalized speed is

$$
F_{r}=\sum_{j=1}^{17}\left(F_{r}\right)^{j}
$$

where $\left(F_{r}\right)^{j}$ is the contribution to the set of holonomic generalized active forces due to the $j^{t h}$ rigid body. That is,

$$
F_{r}={ }_{l} Q_{r}^{F}+\sum_{i=1}^{8}{ }_{l} Q_{r}^{A_{i}}+\sum_{i=1}^{8}{ }_{l} Q_{r}^{P_{i}}
$$

where $A_{i}$ and $P_{i}$ represent the arms and push-rods, respectively. Likewise the contribution to the set of holonomic generalized inertia forces is

$$
F_{r}^{*}=\sum_{j=1}^{17}\left(F_{r}^{*}\right)^{j}=\left({ }_{l} Q_{r}^{*}\right)^{F}+\sum_{i=1}^{8}\left({ }_{l} Q_{r}^{*}\right)^{A_{i}}+\sum_{i=1}^{8}\left({ }_{l} Q_{r}^{*}\right)^{P_{i}} .
$$

The expressions in Equations 12 and 13 are developed from velocities, accelerations, and angular velocities of the various points and bodies of ARIS used in Equations 9 and 10. Because for ARIS these quantities are typically 20 times as long in nonlinear as in linearized form, the effect of linearization is quite dramatic.

\section{LINEARIZED REDUCED-MASS MODEL}

A further simplification can be made by setting the mass properties (masses and inertias) of the actuator arms and push-rods to zero, while retaining those of the ISPR. The arms and push-rods represent 16 of the 17 bodies in the generalized inertia forces of the nonlinear and linearized models, but only about 1.5 percent of the mass of an ARIS-outfitted ISPR. By setting the mass properties of the arms and push-rods to zero, the number of expressions in the set of generalized inertial forces (see Eq. 13) is reduced by $288(r=1, \ldots, 48, i=$ $1, \ldots, 16)$. If one develops the relationship between the dependent and independent generalized speeds in the form of Equation 3, the nonholonomic and holonomic generalized inertia forces are related to each other as follows:

$$
\tilde{F}_{r}^{*}=F_{r}^{*}+\sum_{s=7}^{48} F_{s}^{*} A_{s r}(r=1, \ldots, 6) .
$$

Since Kane's method yields only as many dynamical equations as independent generalized speeds, removing these 288 expressions from the holonomic generalized inertial forces produces a net reduction of 1728 $(=6 \times 288)$ terms in the final system equations.

Despite the great reduction in number of terms in the generalized inertia forces, the simplified (reducedmass) dynamical equations maintain the integrity of both the generalized active forces (including control and disturbance inputs) and the constraint equations. The final dynamical equations, therefore, are the same as those reported previously by Hampton and Beech ${ }^{5}$, excluding the contributions to the generalized inertia forces from the arms and push-rods.

\section{COMPARISON OF FULL NONLINEAR MODEL TO TRUTH MODEL}

To compare the truth model and the nonlinear model, the upper and lower stingers and cross-flexure spring stiffnesses (internal actuator forces) were set to negligible levels, $0.001 \mathrm{ft}-\mathrm{lbf} / \mathrm{rad}(0.00021 \mathrm{in}-$ lbf/degree). Various test inputs were applied, at selected "target points," to compare these models. Two such test inputs were (1) a constant, one-pound force with a constant direction relative to the ISPR; and (2) an oscillating force with an amplitude of one-pound and an angular frequency of 0.2 radians per second. For the tests presented below, the target point was arbitrarily displaced six inches in each orthogonal (coordinateaxis) direction from the flotor center of mass. The direction of application for each force is along the ISPR $x$-axis.

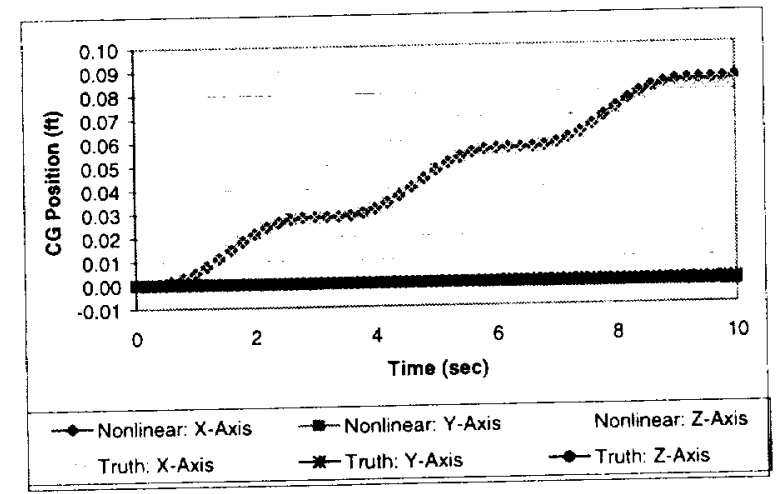

Figure 4. Comparison \#l Between Truth- and Nonlinear Models

The first comparison between the nonlinear and truth models (Fig. 4) shows the response of each model to an oscillating input force applied directly to the ISPR. The respective model behaviors are very similar 
throughout the ten-second simulation. The differences between the two can be attributed to numerical roundoff errors in the fixed rotations between the ISS-, ISPR-, and actuator-fixed coordinate systems in the full nonlinear model, and some minimal effect due to the actuator springs. In this comparison, the actuators are not fully extended, so there are no effects from the constraint equations.

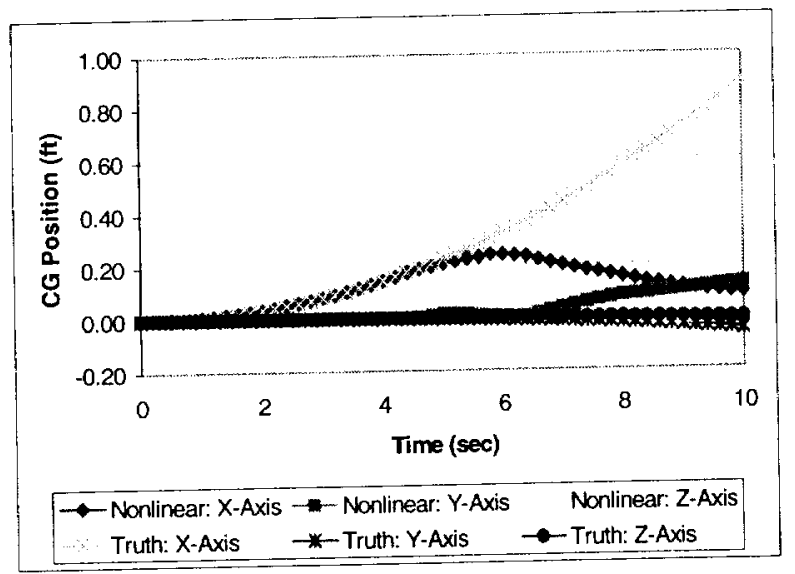

Figure 5. Comparison \#2 Between Truth- and Nonlinear Models

The second comparison (Fig. 5) shows the response of each model to a constant input force applied to the ISPR. In this model, the response of the nonlinear model tracks the response of the truth model for about 4.4 seconds, or until the mass center (CG) has moved approximately three inches $(0.25 \mathrm{ft})$. At this point, an actuator linkage is fully extended, so that it jerks the ISPR, causing its motion to reverse direction.

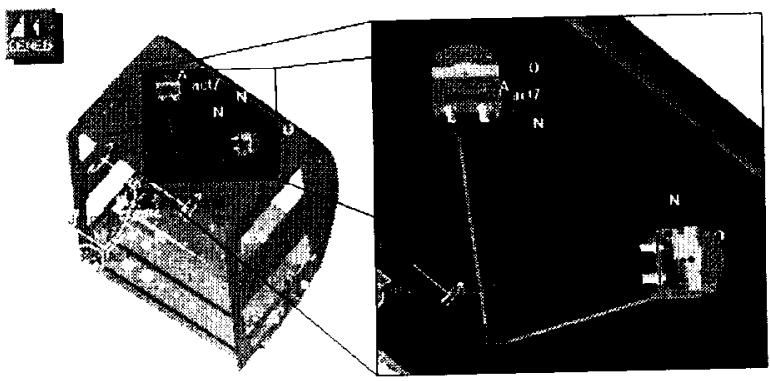

Figure 6. Envision Visualization of ARIS Actuators Near Full Extension

This explanation for the observed behavior was confirmed by the visualization afforded by the Envision model. In the AUTOLEV simulation, at the time when the ISPR center of gravity reversed direction, namely 4.4 seconds, the actuator configuration predicted by AUTOLEV was recorded. That data was then entered into the Envision model, which clearly showed an actuator arm and push-rod having just reached full extension (see Fig. 6). These two simulations indicate that if the simple truth model is to be believed, so also is the full nonlinear model.

\section{COMPARISON BETWEEN LINEARIZED AND NONLINEAR MODELS}

The small-angle theorem is based on a truncated Maclaurin-series expansion of the expressions for sine and cosine (about 0 ):

and

$$
\begin{aligned}
& \sin \theta=\theta-\frac{\theta^{3}}{3}+\frac{\theta^{5}}{5}-\ldots, \\
& \cos \theta=1-\frac{\theta^{2}}{2}+\frac{\theta^{4}}{4}-\ldots .
\end{aligned}
$$

Depending on the application, this approximation is accurate for values of $\theta$ less than about 17 degrees. In the present application, the linearization is particularly relevant to the constraint equations; values of $\theta$ less than about eight degrees produce small-angle approximations that stay within the error tolerance for the encapsulated constraint equations.

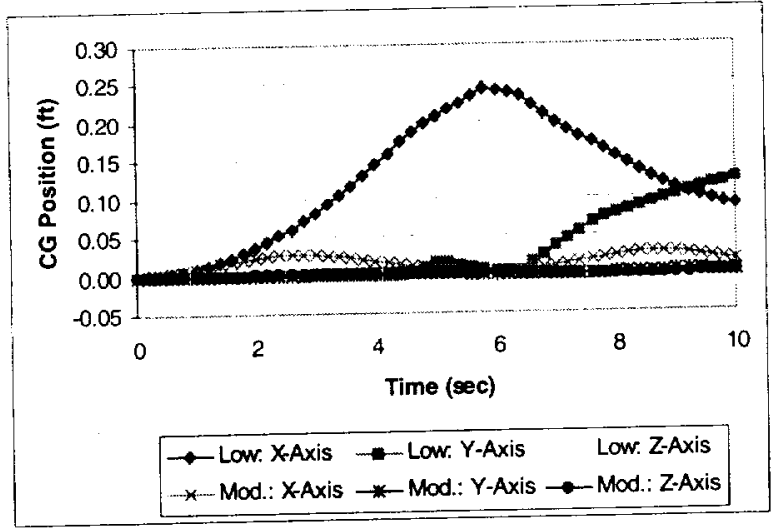

Figure 7. Low vs. Moderate Actuator Spring Stiffness, Response to Constant Test Input

To ensure that the ISPR remains within its rattlespace (for open-loop simulation purposes), so that the small-angle approximations remain valid, the spring stiffnesses at the cross-flexure and upper and lower stingers were increased to nominal levels. The spring rates were set to $1.0 \mathrm{ft}$-lbf/rad $(0.21 \mathrm{in}-\mathrm{lbf} /$ degree $)$. In Figure 7, one can see how the effect of the increased actuator spring constants changes the ISPR response to the applied force. The ISPR motion in the case of moderate actuator spring constants is relatively small, compared to the ISPR motion for negligible actuator spring constants. 


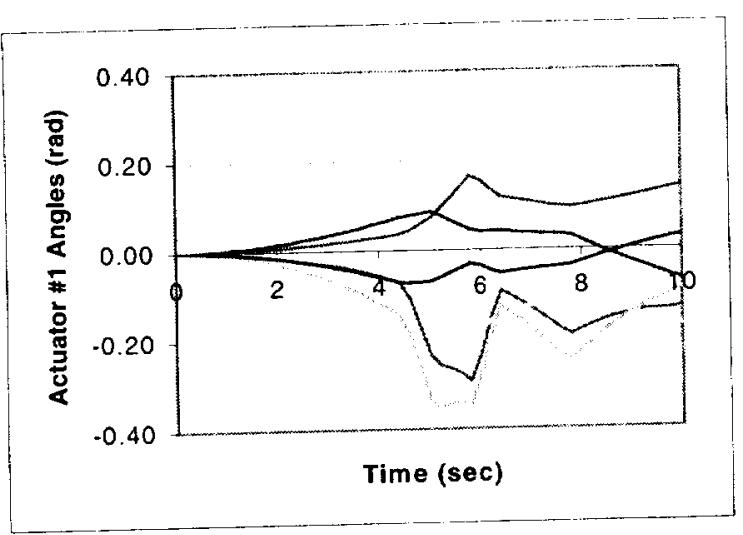

Figure 8. Actuator \#1 Angles, for Low Actuator Spring Rates

Figure 8 shows the response, measured in actuator \#l angles, when the spring constants are negligible and the ISPR is subjected to a constant input force. At about 4.4 seconds, the actuator angles exceed the region bounded by the $+/-0.2$ radians, within which the linearized constraint equations are valid. Accordingly, Figure 9 which shows that the linearized model corresponds well to the nonlinear model until the simulation reaches 4.4 seconds. After that time, the linearized model fails because the constraint equations no longer hold.

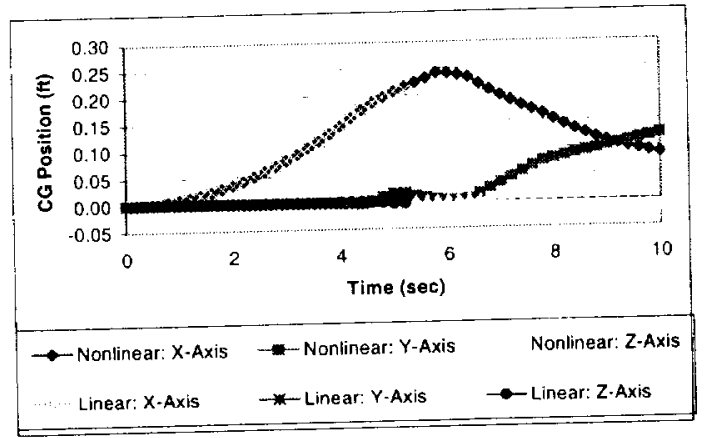

Figure 9. Linearized vs. Nonlinear Model, for Low Stiffness: Response to Direct, Constant Test Input

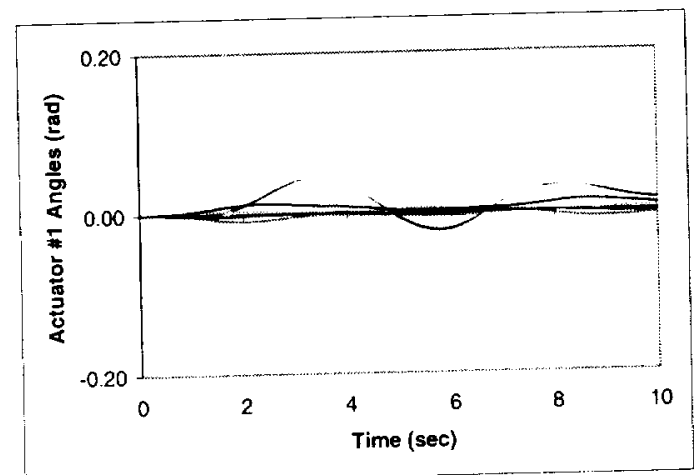

Figure 10. Actuator \#1 Angles, for Moderate Actuator Spring Rates

Figure 10 shows the response, measured in actuator \#1 angles, when the spring constants are increased to moderate levels (0.21 in-lb/degree) and the ISPR is again subjected to a constant input force. In this case, the angles never exceed the region defined by $+/-0.20$ radians. In fact, the angles for actuator \#1 never exceed +1 - 0.10 radians. Figure 11 shows a comparison between the ISPR motion for the linearized and nonlinear models when the actuator spring constants are increased to moderate levels. In this case there is nearly an exact match between the two models.

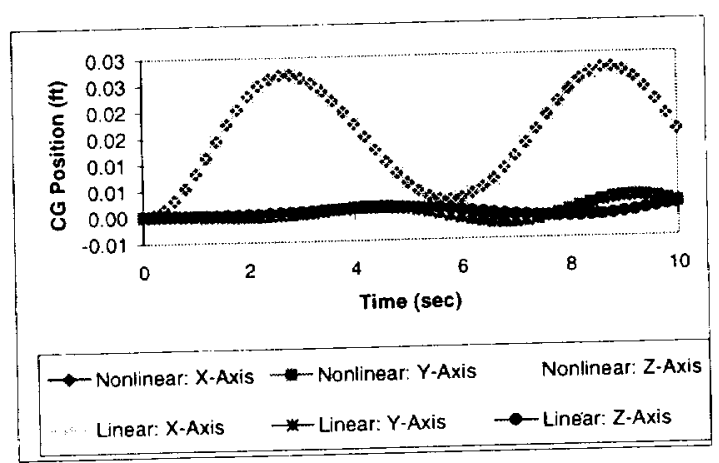

Figure 11. Linearized vs. Nonlinear Model, for Moderate Stiffness: Response to Direct, Constant Test Input

This is an important result; it shows that for small angles, the linearized model very closely tracks the full nonlinear model, which, as was noted previously, tracks the truth model. With the actual hardware, the ISPR will stay centered in its rattle-space due to the active control system. Assuming, then, the correctness of the simple truth model, the linearized model is accurate within operational (rattle-space) limits.

\section{COMPARISON BETWEEN LINEARIZED REDUCED-MASS AND NONLINEAR MODELS}

Since the generalized inertia forces are the most complex expressions in the dynamical equation development, the omission of the 288 expressions for the arms and push-rods represents a substantial reduction in the overall size of the dynamical equations, as noted before (see Eqs. 10,13). This simplification is made by

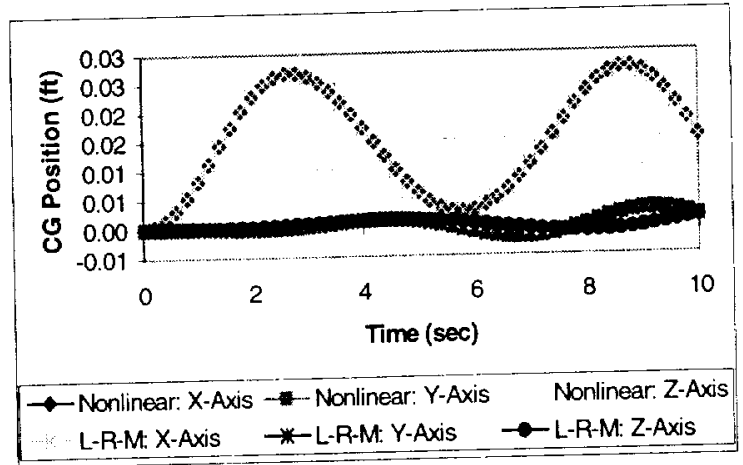

Figure 12. Linearized Reduced-mass vs. Nonlinear Model, for Moderate Stiffness: Response to Direct, Constant Test Input 
by simply setting the appropriate masses, and the appropriate moments and products of inertia, to zero in the AUTOLEV code, and then recompiling the generated C program.

Figure 12 shows the response measured, in linear displacement of the ISPR center of mass, when the spring constants are increased to moderate levels $(0.21$ in-lbf/degree) and the ISPR is again subjected to a constant input force. From this plot, it is apparent that neglecting the mass properties of the arms and push-rods has little effect on the model's description of system behavior. This is as expected, for two reasons. First, as noted previously, the masses of the arms and push-rods are relatively small in comparison to the overall mass of an ARIS-outfitted ISPR. Second, because the ARIS arms and push-rods are more closely linked to the (relatively "fixed") space station than to the ISPR, the amplitudes of their displacements, velocities and accelerations will be correspondingly smaller than those of the ISPR.

\section{CONCLUSION}

This paper has presented comparisons among nonlinear, linearized, and linearized reduced-mass models of ARIS (all without umbilicals). Direct and indirect disturbances were applied to these three models, and the responses were compared. It was concluded the linearized reduced-mass model captures the significant system rigid-body dynamics.

Anticipated future work includes (1) the implementation of the linearized, reduced-mass model, including umbilicals, in MATLAB; (2) model improvements based on actuator-characterization and on-orbit system characterization experiments; and (3) use of the MATLAB model for optimal controller design.

\section{REFERENCES}

1. DeLombard, R., Bushnell, G.S., Edberg, D., Karchmer, A. M., and Tryggvason, B. V., "Microgravity Environment Counter-measures Panel Discussion," AIAA 97-0351, January 1997.

2. Edberg, D., Boucher, R., Schenck, D., Nurre, G., Whorton, M., Kim, Y., and Alhorn, D., 1996, "Results of the STABLE Microgravity Vibration Isolation Flight Experiment," Guidance and Control, Vol. 92, pp. 567-581.

3. Whorton, M. S., March 1998, "g-LIMIT: A Vibration Isolation System for the Microgravity Science Glovebox," presented to the $17^{\text {th }}$ Microgravity Measurements Group Meeting.
4. "System Specification for the International Space Station," Specification Number SSP41000, Rev. D, NASA Johnson Space Center, November 1, 1995.

5. Beech, G. S., A 'Kane's Dynamics' Model for the Active Rack Isolation System, Masters Thesis, University of Alabama in Huntsville, Dec. 2000.

6. Kane, T. R., and Levinson, D. A., Dynamics: Theory and Applications, McGraw-Hill, Inc., New York, 1985.

7. Rupert, J. K., and Hampton, R. D., "An Improved Umbilical Model for The Active Rack Isolation System," AIAA-2000-0573, January 2000.

8. Kane, T. R., and Levinson, D. A., Dynamics Online: Theory and Implementation with AUTOLEV $V^{T M}$, Thomas R. Kane and David A. Levinson, 1996. 